\title{
Mucoadhesive Pectin-Based Cross-Linked Microgels
}

\author{
Dmitrii A. Tolstykh, Ksenia V. Kozhikhova, Maxim A. Mironov \\ Department of Technology for Organic Synthesis, Ural Federal University \\ Mira 28 St., Ekaterinburg, Russia \\ d.a.tolstykh@gmail.com; k.v.kozhikhova@gmail.com; m.a.mironov@urfu.ru
}

\section{Extended Abstract}

Polysaccharide-based microgels possess the great potential for drug delivery application. They have a large surface area with high amount of functional groups able for bioconjugation [1]. Microgels can be obtained by either chemical or physical cross-linkage methods. Our research group elaborated the approach for synthesis of cross-linked polysaccharide microgels via the Ugi multicomponent condensation (U-4CR) in diluted colloidal suspensions (at polysaccharide concentrations below the critical concentration of chain entanglement) [2].

The objective of this research was to develop the new range of microgels suitable for drug delivery application. Modified pectin comprising hydrazyde groups has been chosen as a polymeric network according to its biocompatibility, biodegradability, high mucoadhesiveness, and possession of both acidic and basic functional groups.

In our research we employed two kinds of modified pectin: with $24 \%$ and $33 \%$ degree of substitution. Polysaccharide chains were cross-linked via U-4CR, which includes interaction of amino, carbonyl, carboxyl and isocyano groups. We used six aldehydes and eight isocyanides for the cross-linkage. The isocyanides quantities were varied between 5 and 20 mol\% to obtain different linkage densities. The modified pectin concentration was $0,1 \%$.

The employment of pectin derivative with lower degree of substitution has resulted in visible macrogel particles formation. The same result has been achieved by the usage of formaldehyde in the reaction. Pectin-based microgel particles with the average hydrodynamic diameter in the range of $210-550 \mathrm{~nm}$ have been synthesized using the modified pectin with $33 \%$ degree of substitution and aldehydes with short aliphatic chains. We have observed that an increase in aliphatic chain length makes positive impact on monodispersity of particles. It can arise from the assembly of hydrophobic cores and increase of intramolecular cross-linking. The chemical structures of the products were confirmed by the ${ }^{1} \mathrm{H}$ NMRspectroscopy. The sizes and polydispersity indexes of particles were determined by dynamic light scattering. Having based on our previous results and the chemical structure, we supposed $\mathrm{pH}$-responsibility of our new range of microgels. By the $\mathrm{pH}$ variation we have found the swollen state of microgels at physiological $\mathrm{pH}$ range and their shrinking in highly acidic media.

Mucoadhesive properties of microgels have been evaluated by the incubation of the microgels with equivalent amounts of mucin and calculation of the mucin adsorption [3]. The amounts of free mucin were determined after centrifugation according to the Bradford method [4]. We have observed that the best mucin affinity is exhibited by the microgel which was synthesized using N-3-isocyanopropyl-N,N-diethylamine and isovaleraldehyde. The highest mucoadhesiveness can be explained by the basic properties of diethylamino moiety.

The cytotoxicity of the microgels has been determined against HeLa cells by the MTT assay [5]. The microgels obtained via cross-linking using isovaleraldehyde and N-3-isocyanopropyl-N,N-diethylamine or 3(isocyanomethyl)pyridine have shown weak cytotoxicity (cell viability within 70-80 \%), the microgel synthesized with $\mathrm{N}$ 3 -isocyanopropyl-N,N-diethylamine and isovaleraldehyde has been evaluated as non-cytotoxic (cell viability above $80 \%$ ).

Furthermore we have developed the method of labeling microgels by fluorescent dye via 4-UCR. We used isocyanide linked with Rodamine B. The fluorescent dye was synthesized via interaction of Rodamine B isothiocyanate with 1-(2methylprop-1-en-1-yl)piperidine and one of the isocyano groups of 1,4-bis(3-isocyanopropyl)pyperazine. The fluorescent label can be employed to determine the distribution of the microgel particles in living organisms.

In summary the new range of mucoadhesive microgels possessing a potential for drug delivery application has been developed and the properties of the microgel particles have been evaluated. 


\section{References}

[1] J. K. Oh, et al., "Biopolymer-based microgels/nanogels for drug delivery applications," Progress in Polymer Science, vol. 34, no. 12, pp. 1261-1282, 2009.

[2] M. A. Mironov, et al., "Synthesis of polyampholyle microgels from colloidal salts of pectinic acid and their application as pH-responsive emulsifiers," Colloid. Polym. Sci., vol. 291, no. 7, pp. 1683-1691, 2013.

[3] F. Qiang, et al., "Enhanced systemic exposure of fexofenadine via the intranasal administration of chitosan-coated liposome," International Journal of Pharmaceutics, vol. 430, no. 1-2, pp. 161-166, 2012.

[4] M. M. Bradford, "A rapid and sensitive method for the quantitation of microgram quantities of protein utilizing the principle of protein-dye binding," Analytical Biochemistry, vol. 72, no. 1-2, pp. 248-254, 1976.

[5] J. M. Chan, et al., "PLGA-lecithin-PEG core-shell nanoparticles for controlled drug delivery," Biomaterials, vol. 30, no. 8, pp. 1627-1634, 2009. 